\title{
Creating Effective Models for Delivering Palliative Care in Advanced Liver Disease
}

\author{
Manisha Verma ${ }^{1} \cdot$ Marie A. Bakitas ${ }^{2,3}$ \\ Accepted: 25 March 2021 / Published online: 10 April 2021 \\ (C) The Author(s), under exclusive licence to Springer Science+Business Media, LLC, part of Springer Nature 2021
}

\begin{abstract}
Purpose of Review The current healthcare system is not fully equipped to provide comprehensive support for patients with advanced liver disease (ALD) and their caregivers resulting in concomitant suffering and reduced quality of life (QoL). Integration of palliative care (PC) within routine care has demonstrated benefits in improving symptoms and QoL and reducing healthcare utilization for other serious illnesses but has been underutilized or delayed for ALD care. The purpose of this article is to outline the domains and benefits of PC and discuss the misconceptions and barriers for PC integration, and healthcare delivery models supporting PC integration within ALD care.

Recent Findings PC has eight key domains related to physical and mental health, goals for future care, and care of the caregivers. PC offers benefits to improve health outcomes and patient satisfaction and reduce healthcare utilization. To date there have been successful models of PC that are primarily hospital- or community-based; successful models have been PC specialist- or primary/ generalist-led.

Summary Concurrent PC within oncology has formed the basis for most evidence-based guidelines. PC integration within ALD care is still in its infancy. While amassing evidence in ALD, hepatology organizations can promote consensus-based integrated PC models that can guide research and practice efforts to increase supportive care for these patients in need and their family caregivers.
\end{abstract}

Keywords Models of palliative care · Advanced liver disease

\section{Introduction}

The prevalence of advanced liver diseases (ALD) has doubled in the past decade globally. This is primarily due to increase in decompensated cirrhosis and hepatocellular cancer (HCC) [1]. ALD is one of the three leading causes of decreased life expectancy in USA [2]. HCC is the 2nd leading cause of cancer-

This article is part of the Topical Collection on Palliative Care

Manisha Verma

VermaM@einstein.edu

1 Department of Digestive Diseases and Transplantation, Einstein Healthcare Network, Philadelphia, PA, USA

2 School of Nursing, University of Alabama at Birmingham (UAB), Birmingham, AL, USA

3 Department of Medicine, Division of Gerontology, Geriatrics and Palliative Care, UAB Center for Palliative and Supportive Care, UAB, Birmingham, AL, USA related deaths globally, with an exponential increase in incidence and mortality rates [3]. These observations are particularly important in the context of stable or falling death rates for heart disease and non-liver cancer, respectively $[4,5]$. The symptom and psychological burden in this population is high, with the most frequent symptoms being pain, breathlessness, muscle cramps, sleep disturbance, and psychological symptoms (depression and anxiety) [6]. This translates into impaired quality of life (QoL), loss of productivity, and increased healthcare utilization [7]. Hospitalization rates due to ALD and its complications skyrocketed by $93 \%$ (due to hepatorenal syndrome), $62 \%$ (due to portal hypertension), and $190 \%$ due to hepatitis C and its complications from 2004-2005 to 2010-2011 [8].

Inevitably, ALD impacts the caregivers, increasing caregiver burden and distress $[9,10]$. Studies on the emotional toll of ALD on patients' caregivers result in caregivers with decreased mental well-being compared to the general population with prevalent depression and anxiety [11]. The current healthcare system has not provided routine comprehensive 
support for patients with ALD and their caregivers resulting in concomitant suffering, reduced QoL, and high healthcare utilization [12]. Importantly, evidence from supportive and palliative care (PC) studies in cancer and non-cancer populations (including ALD) suggest that this critical care gap can be bridged by incorporating PC services throughout the liver disease trajectory $[13,14,15]$. The purpose of this article is to outline PC key domains and benefits, misconceptions and barriers for PC within ALD care, and delivery models that support PC integration within routine ALD care.

\section{Palliative care: Definition, Domains, and Outcomes}

Palliative care is a medical specialty, delivered by an interprofessional team to patients and their family members, aimed at improving symptom control, QoL, illness understanding, and decision-making [16]. Over the past 2 decades, the evidence base supporting PC inclusion in serious illnesses such as cancer, heart failure, and stroke has expanded such that now multiple specialty organizations recommend concurrent PC as a care standard $[17,18]$. These guidelines generally recommend some elements of PC be provided early in the disease course and continue to be delivered concurrent with disease-directed therapies. The quantity and characteristics of PC may wax and wane over time, with respect to patient and family needs (Fig. 1). At earlier stages of disease or when symptom burden is low, PC can be helpful in assisting patients and family members to learn skills to help them to cope with current or future physical and other stresses of serious illness and how to make valuebased decisions about treatments and advanced care planning. While at later stages of disease or when symptom burden increases, PC can help with symptom control, goals of care planning/implementation, and identifying preferences for culturally consistent end of life care. After death, family bereavement support can continue.

The National Consensus Project, Clinical Practice Guidelines for Quality Palliative Care - 4th edition identifies eight domains of care $[13,19]$ :

1. Structure and processes of care: The composition of an interdisciplinary team is outlined, including the professional qualifications, education, training, and support needed to deliver optimal patient- and family-centered care. This also defines the elements of the PC assessment and care plan, as well as systems and processes.

2. Physical aspects of care: Describes the PC assessment, care planning, and treatment of physical symptoms emphasizing patient- and family-directed holistic care.

3. Psychological and psychiatric aspects of care: Focuses on the processes for systematically assessing and addressing the psychological and psychiatric aspects of care in the context of serious illness.

4. Social aspects of care: Outlines the PC approach to assessing and addressing patient and family social support needs.

5. Spiritual, religious, and existential aspects of care: Describes the spiritual, religious, and existential aspects of care, including the importance of screening for unmet needs.

6. Cultural aspects of care: Outlines the ways in which culture influences both PC delivery and the experience of that care by the patient and family, from the time of diagnosis through death and bereavement.

7. Care of the patient nearing the end of life: This domain focuses on the symptoms and situations that are common in the final days and weeks of life.

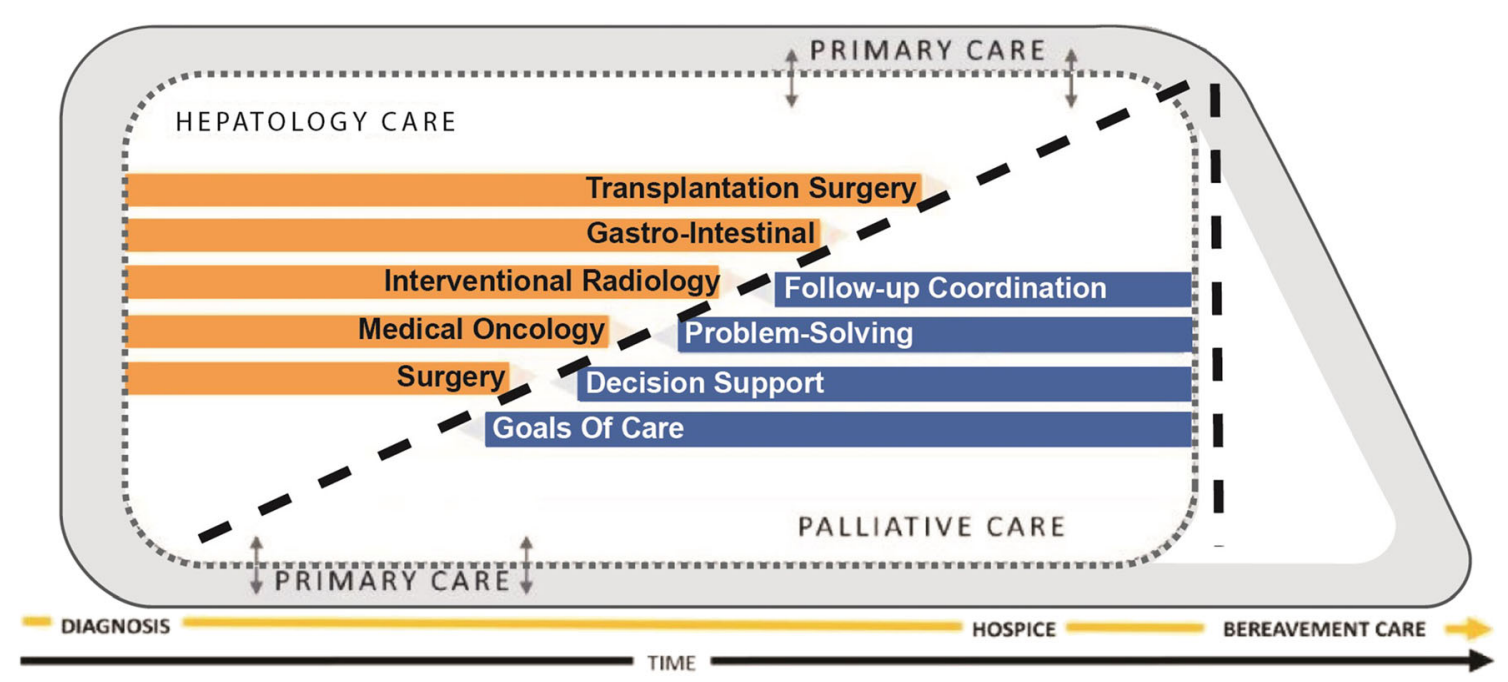

Fig. 1 A proposed model of concurrent palliative care for ALD, including transplant evaluation 
8. Ethical and legal aspects of care: Content includes advance care planning, surrogate decision-making, regulatory and legal considerations, and related PC issues, focusing on ethical imperatives and processes to support patient autonomy.

PC has been shown to reduce healthcare costs, improve the quality of care, and align the goals of care between patients/ families and their providers [20]. PC improves health outcomes and QoL in patients with cancer [21]. A comparison of early versus delayed PC integration shows that early integration offers additional benefits to patients including reduced healthcare utilization and 30 day mortality [22]. Specialized PC interventions in different settings have demonstrated such benefits for serious illnesses like heart failure, lung and other advanced cancers, and multiple sclerosis [23, 24].

Coping, treatment decision-making, and advance care planning are the key elements linked with improved patient outcomes. A landmark study for patients with early stage cancer showed that patients who had higher proportion of visits regarding coping showed improved QoL $(p=.02)$ and depression $(p=.002)$; patients who had higher proportion of visits on treatment decisions were less likely to initiate chemotherapy or hospitalization [25].

\section{Misconceptions and Barriers to integrated Palliative and Advanced Liver Disease Care}

Palliative care is underutilized in patients with ALD. Over the past decade, research has been ongoing to identify prominent misconceptions and barriers in order to define strategies to overcome them. First, PC is often equated to hospice or end of life care by both patients and providers. This points to the need for both patient and provider education [26]. Second, hepatologists believe that their goal is to get the patient to transplant, and if they introduce PC to patients during a transplant evaluation, it may give the patient the sense that they are giving up on them or that they might be dying [27]. This points to the need for demonstration projects that disprove the false claim of giving up. Third, gastroenterologists and hepatologists often lack training in primary PC skills (such as communication, caregiver support, psychological care) which limits their ability to provide this type of support to their patients. This points to need for development and inclusion of primary PC skills training programs within GI/ hepatology fellowship training. Fourth, inadequate access to PC due to limited workforce and resources has become even more critical, as the demand for PC services within cancer and non-cancer serious illnesses has increased. This supports the need to provide greater education to hepatologists and gastroenterolgists about PC. Fifth, lack of clear criteria on when and who should be referred to PC. This points to the need of creating screening checklists for ALD, informed with algorithms to follow when PC can be triggered.

An active area of investigation in PC includes defining the most critical PC components, and who are the providers of $\mathrm{PC}$ ? As mentioned, the growing demand for PC provided by a team comprised of board-certified physicians, nurses, social workers, and chaplains will not be able to meet the needs of all who could benefit from this care approach. Hence a "tiered" approach of primary (also called generalist) and specialist PC has been proposed [28]. Primary PC involves all healthcare professionals, such as hepatologists, who are not PC specialists but have undergone some training in basic PC principles of symptom control, communication about prognosis, goals of treatment, and life-sustaining treatments, whereas specialty $\mathrm{PC}$ is provided by an interprofessional team that has had years of specialty training and are board-certified.

Although it may seem appropriate to refer all ALD patients to a PC provider, this is unlikely to be feasible, scalable, or necessary. First, the PC providers will be overburdened to meet the demand, adding to existing shortage of PC providers [29]. Second, adding another specialist within care of already complex patients may unintentionally undermine existing therapeutic relationships [30]. Third, non-PC providers may defer symptom management to a PC provider, who may be less comfortable in some approaches, such as medications, due to the underlying liver disease. These reasons support the development of a sustainable model, involving formal training of gastroenterologists and hepatologists to offer PC and equipping them with baseline competency and resources to render effective and expedient PC. Table 1 lists a potential training program for $\mathrm{GI} /$ hepatologists to learn the skills of primary PC that can assist them to provide this for a large portion of their patients and reserving referral to specialists for the most complex or refractory cases. This training program has demonstrated improvement in communication skills, psychosocial support, goals of care discussions, and caregiver support [31].

\section{Healthcare Delivery Models of Integrated Palliative Care}

A model of care is defined as "a descriptive picture of practice which adequately reports the real thing." [32•] PC is by definition comprehensive supportive care throughout the disease trajectory. Multiple methods of delivering PC have been tested and found to be effective. Unfortunately, this heterogeneity of PC models and inconsistent reporting of the components, methodological limitations, and lack of consensus on outcome measures has made it difficult to compare and contrast different approaches $[33,34 \bullet \bullet]$. Limited research has been done on models of care for ALD. 
Table 1 Core competencies of a gastroenterologist/hepatologist training program

\begin{tabular}{|c|c|}
\hline Learning modules & Core skills \\
\hline Module 1: Introduction to palliative care & $\begin{array}{l}\text { Describe the scope and role of palliative care as part of an interdisciplinary patient and family care plan. } \\
\text { Understanding the holistic concept of PC and how it is inclusive of supportive care from the onset of } \\
\text { disease through the disease trajectory, including end of life care }\end{array}$ \\
\hline $\begin{array}{l}\text { Module 2: Communicating with patients and } \\
\text { families }\end{array}$ & $\begin{array}{l}\text { Practice effective communication skills with patients, families, and other healthcare providers, including } \\
\text { responding to emotion, coaching in self-management of symptoms and distress. Applying motivational } \\
\text { interviewing techniques to communication }\end{array}$ \\
\hline Module 3: Psychosocial support & Conduct regular assessment of psychosocial needs using instruments such as distress thermometer \\
\hline $\begin{array}{l}\text { Module 4: Discussing goals of care and } \\
\text { advance care planning }\end{array}$ & $\begin{array}{l}\text { Describe the issues around transitions in care (e.g., transplant eligibility, transition to hospice) and key } \\
\text { needs for patients and caregivers at these times. Developing goals of care and advance directives to } \\
\text { document the decisions made }\end{array}$ \\
\hline $\begin{array}{l}\text { Module 5: Symptom management: physical } \\
\text { symptoms and pain }\end{array}$ & $\begin{array}{l}\text { Conduct regular assessment of physical symptoms using instruments such as Edmonton Symptom } \\
\text { Assessment Scale, and apply evidence based medicine to manage the symptoms }\end{array}$ \\
\hline $\begin{array}{l}\text { Module 6: Symptom management: } \\
\text { psychological symptoms }\end{array}$ & $\begin{array}{l}\text { Conduct regular assessment of psychological symptoms using instruments such as depression screening, } \\
\text { and apply evidence based medicine to manage them }\end{array}$ \\
\hline Module 7: Spiritual care & $\begin{array}{l}\text { Understanding the role of spirituality within medical care approaches; benefits of involving chaplains } \\
\text { when needed }\end{array}$ \\
\hline Module 8: Hospice care & Overview of hospice and when to refer patients for hospice services \\
\hline Module 9: Survivorship & Enabling and empowering patients post-transplant and those living with cancer \\
\hline Module 10: Care of the caregiver & Respond to common caregiver needs throughout the care continuum \\
\hline
\end{tabular}

We utilized the research and exemplars from cancer- and non-cancer-integrated PC models to outline the features that might be applicable to an ALD population. We describe the PC models under two main groups: (A) specialty PC delivered by board-certified providers and (B) primary (or generalist) palliative care delivered by providers who are trained on certain basic principles of PC. These differ in their team structures, care processes, location of care, and related reimbursements.

In addition, the consensus recommendations for operational features of PC models (based on the National Quality Forum) are outlined in Table $2[35,36]$.

\section{Specialist PC Models (Table 3)}

\section{Hospital-based PC (HBPC)}

The growth of PC services within US hospitals is largely due to the evidence of its value in improving care especially for the high volume of high acuity, seriously ill inpatients. According to a state-by-state report card, as of $2019,72 \%$ of hospitals with greater than 50 beds report having a PC team [37]. Hospital-based PC generally consists of a consultation service and in some cases an inpatient specialty PC unit. PC teams are typically comprised of board-certified PC physicians, advance practice nurse practitioners, social workers, chaplains, volunteers, and psychologists. While all interprofessional PC team members' salaries may not be officially supported within a PC department, availability of these clinicians is critical to the success of the team. PC providers may provide consultation only or may also assume a primary role for care coordination.

Inpatient consults for PC have been shown to reduce symptom burden and enhance satisfaction with care, while simultaneously reducing costs by aligning therapeutic decisions with patients' preferences and values. Code status discussions improved after PC consultations. A large multicenter national collaborative of PC teams across healthcare organizations in the USA ( 88 teams from 17 states) demonstrated enhanced goals of care discussions and thereby improved goal concordant care. There was $18 \%$ change in preferences for DNR/ DNI after HBPC consults [38]. In fact, the percentage of patients discharged home alive increased and referrals to hospice decreased over time, suggesting that PC consults are trending toward increased effectiveness in safe home tran-

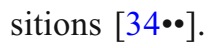

A study of patients with hematopoietic stem cell transplantation demonstrated improved quality of life, reduced anxiety/depression, and better symptom control in inpatients who received PC consults [39]. Caregivers reported lower rates of depression at 6-month post-transplant. This important study demonstrates the benefit of introducing PC within curative care. Similar benefits may be applicable for ALD inpatients being considered for or during admission for liver transplantation. There is a high need for developing and testing such interventions in multiple transplant centers.

The REDAPS (Randomized Evaluation of Default Access to Palliative Services) trial is a pragmatic stepped-wedge 
Table 2 Consensus Recommendations for operational features of palliative care (PC) programs

Domain Features $\quad$ ALD-specific features

\section{Program administration (mission alignment of PC program with institutional aims to improve patient centered care) \\ 2. Type of services (inpatient, outpatient, home based, telehealth)}

3. Availability (routine and emergency)

4. Staffing (physicians, nurses, social work, chaplain)

5. Measurement

6. Quality improvement (QI)

\section{Marketing}

8. Education

9. Bereavement services

10. Patient identification

11. Continuity of care

12. Staff wellness
PC program staff is integrated into the management structure of the hospital and practice to align the mission and values.

Needed: (1) a designated program director, (2) reporting mechanism in place

A consultation service that is available to all inpatients; access to outpatient PC services and an inpatient PC unit

24/7 access to PC providers for inpatients; access to outpatient clinics during the week, 24/7 telephone/ telehealth access, appointments available within 24-48 $\mathrm{h}$

Specific funding for a PC provider (board certified), PC certified nurse, trained staff to provide mental health services. Social work and administrative support staff

Key outcome measures:

- Operational metrics (\# of consults, referring physicians, disposition)

- Clinical metrics: improvement in pain, dyspnea, and distress

- Customer metrics (patient/family/referring physician satisfaction with $\mathrm{PC}$ service)

- Financial metrics: cost avoidance, billing revenue, length of stay

Quality improvement activities, continuous or intermittent for (a) pain, (b) non-pain symptoms, (c) psychosocial/spiritual distress, and (d) communication between healthcare providers and patients/surrogates

Marketing materials and strategies appropriate for hospital staff, patients, and families

PC educational resources for hospital physicians, nurses, social workers, chaplains, trainees, and any other staff the program feels are essential to fulfill its mission and goals

A bereavement policy and procedure that describes bereavement services provided to families of patients affected by the PC program

Make changes as needed through QI initiatives. Telephone or letter follow-up, sympathy cards, registry of community resources for support groups and counseling services, and remembrance services

A working relationship with the appropriate departments to adopt PC screening criteria for patients in the emergency department, general medical/surgical wards, and intensive care units

Policies and procedures that specify the manner in which Coordination of care as ALD patients move from one care transitions across care sites (e.g., hospital to home hospice) will be handled to ensure excellent communication between facilities. A working relationship with one or more community hospice providers

Policies and procedures that promote PC team wellness. The psychological demands on PC staff are often overwhelming, placing practitioners at risk of burnout or other mental health problems
- Transplant hepatologists include PC providers within their management structure

- Multidisciplinary care model for ALD includes PC providers

- ALD inpatients are consulted for PC as a part of routine care

- ALD patients getting discharged get an outpatient/telehealth-based follow-up PC appointment

PC providers dedicated to liver service line, who can be contacted for consults

Hepatology department budgets to be inclusive of funds to support PC services

Operational metrics for all ALD consultations. Customer, clinical, and financial metrics that are tracked either continuously or intermittently

QI within ALD care through research to assess the impact of PC services on symptoms, distress, and communication

As an evolving specialty within ALD care, the PC program is responsible for making its presence and range of services known to the key stakeholders for quality care

PC providers help develop educational opportunities and resources to improve the attitudes, knowledge, skills, and behavior of all health providers involved within ALD care

PC providers and hepatologists are required to offer an initial bereavement support for ALD caregivers

To facilitate referrals for "at-risk"

ALD patients," ALD providers adopt screening tools within routine care site to another becomes a standard part of care

Regularly scheduled patient debriefing exercises within transplant care

Relaxation-exercise training and referral for staff counseling cluster randomized trial to assess the efficacy of PC services for inpatients $>65$ years old with advanced lung disease, dementia, or end-stage renal disease. Hospitals are the units of randomization. The primary outcomes of this trial are inpatient mortality and length of stay. The results are awaited [40]. 
Table 3 Models of palliative care: strengths and weaknesses (adapted from Verma M 2020)

\begin{tabular}{|c|c|c|c|}
\hline Model & Description & Strengths & Weaknesses \\
\hline Hospital-based PC & $\begin{array}{l}\text { PC team consulted } \\
\quad \text { during hospitalization }\end{array}$ & $\begin{array}{l}\text { 1) Identified high need population } \\
\text { 2) Helps reduce healthcare utilization, and develop end } \\
\text { of life care goals }\end{array}$ & $\begin{array}{l}\text { 1) Limited continuity of care } \\
\text { 2) Usually it's too late for the patients to } \\
\text { receive the plethora of benefits of PC } \\
\text { 2) Limited number of PC providers available } \\
\text { for high-needs inpatients }\end{array}$ \\
\hline $\begin{array}{l}\text { Outpatient PC } \\
\text { specialty clinics }\end{array}$ & $\begin{array}{l}\text { PC providers (MD, NP, } \\
\mathrm{RN} \text { ) conduct } \\
\text { standalone } \mathrm{PC} \text { clinics }\end{array}$ & $\begin{array}{l}\text { 1) Continuity of care is easily established } \\
\text { 2) Centralized services } \\
\text { 3) Allows for more day-to-day planning and resource } \\
\text { allocation } \\
\text { 4) Autonomy around concise and consistent referral } \\
\text { criteria } \\
\text { 5) Hub for education and research in PC }\end{array}$ & $\begin{array}{l}\text { 1) Startup costs, overhead, and budgetary } \\
\text { implications to be considered to launch } \\
\text { these clinics } \\
\text { 2) Need for additional support staff in the } \\
\text { clinic } \\
\text { 2) Scheduling challenges may be unforeseen } \\
\text { due to the high volume of patients, but } \\
\text { limited providers in these PC clinics }\end{array}$ \\
\hline $\begin{array}{c}\text { Community-based: } \\
\text { home-based PC }\end{array}$ & $\begin{array}{l}\text { PC providers conduct } \\
\text { home visits and } \\
\text { deliver PC at patient's } \\
\text { home }\end{array}$ & $\begin{array}{l}\text { 1) Comfort at home is maintained, while PC continues } \\
\text { 2) Increased satisfaction with care } \\
\text { 3) Reduced hospitalizations and ED visits } \\
\text { 4) More at-home deaths }\end{array}$ & Limited availability and coverage \\
\hline $\begin{array}{l}\text { Community-based: } \\
\text { telehealth-based } \\
\text { PC }\end{array}$ & $\begin{array}{l}\text { Utilization of remote } \\
\text { technology to deliver } \\
\text { PC }\end{array}$ & $\begin{array}{l}\text { 1) PC providers can deliver care to patients irrespective } \\
\text { of the distance, and patient's willingness to return to } \\
\text { clinics for additional appointments } \\
\text { 2) Videoconferencing provides a glimpse into the } \\
\text { homes and social contexts of patients, making PC } \\
\text { more informed }\end{array}$ & $\begin{array}{l}\text { 1) Reimbursement is challenging and varies } \\
\text { across states } \\
\text { 2) Relies on technology, and is limited to } \\
\text { those with access to the Internet }\end{array}$ \\
\hline
\end{tabular}

Verma, M., Tapper, E. B., Singal, A. G., \& Navarro, V. (2020). Nonhospice Palliative Care Within the Treatment of End-Stage Liver Disease. Hepatology, 71(6), 2149-2159

Some HBPC programs also have a dedicated inpatient PC unit, in addition to a consultation team [41]. These acute care units are mostly for patients with uncontrolled symptoms and other complex care needs which can be best managed by the entire PC team. These units provide support to family members as well. Numerous studies have identified that HBPC often helps reduce overall inpatient care costs. An inpatient PC unit helped reduce daily hospital costs by $74 \%$ when compared to usual inpatient care [42].

Another study identified that on average US hospitals could reduce healthcare costs by around $\$ 3$ million per year using a HBPC team [43].

\section{Outpatient PC Specialty Clinic Models}

Outpatient specialty PC clinics have made an important place within oncology care and are the main setting for patients to be seen from diagnosis throughout their disease trajectory. More than $90 \%$ of National Comprehensive Cancer Network (NCCN) cancer centers have these clinics [44]. Outpatient PC models may include a specialty geographically based outpatient clinic or an embedded clinic. An embedded clinic model usually has a PC provider co-located within a primary/specialist care location (e.g., embedded within a cancer or heart failure specialty clinic). A large cluster randomized trial in Canada compared the outpatient specialist PC model with standard of care for patients with advanced cancer [45]. QoL improved in the PC arm; symptom burden, patient satisfaction, and communication also favored PC arm. A meta-analysis reported the benefits of outpatient PC at an early stage of disease [46].

However, the structure of outpatient PC clinics varies widely across the USA. A survey report from the NCCN cancer center shows that of the 20 clinics surveyed, $43 \%$ had both physicians and advance practice providers, $18 \%$ physicians only, $10 \%$ advance practice providers only, and $29 \%$ had other staff, with an average of 3.3 full-time clinicians [47]. The number of patients served by these clinics has progressively increased over time [48]. Several different criteria are utilized to identify who should be referred to these clinics. Some believe it should be all patients with cancer, while more recently needs based criteria have been proposed [49].

\section{Community-Based Specialty Models (Home-based and Telehealth-based)}

Specialty PC, delivered in non-hospital settings, is generally considered community-based. There are a variety of methods of delivering community-based specialty PC. However, 
telehealth and home visit programs are most common [50].The first published trial of a community-based, telehealth model was for patients with cancer in rural New England. The ENABLE (Educate, Nurture, Advise, Before Life Ends) model comprised a specialty in-person outpatient consultation by a board certified physician or nurse practitioner which were then followed by structured phone sessions by a specially trained palliative care nurse practitioner or registered nurse. Two RCTs of this model in cancer demonstrated improved QoL, mood, symptom relief, and overall patient survival [51, 52••]. Family caregivers in the second RCT demonstrated improved caregiver mood and decreased burden [53].

While numerous examples of community PC have been tested and found effective in cancer and non-cancer illnesses, the coronavirus (COVID-19) pandemic led to broad-based adoption of PC by telehealth in a matter of a few weeks [54]. Telehealth has recently taken much more role under the COVID-19 pandemic situation, when all healthcare has shifted to remote options using technology. Providers are able to provide education, support, and symptom management. It has been shown to improve access and minimize acute care visits [55].Telehealth-assisted home-based PC has demonstrated reduced functional decline as early as 2 weeks post intervention, when compared to standard of care (i.e., no PC intervention) ( -1.35 vs. $-12.30(p=0.067)$ [56]. The study population was primarily elderly with an average survival of 5.8 months. Telehealth has been recently utilized for inpatient PC as well, primarily to reduce exposure of providers and save the personal protective equipment for required in person contacts [57].

Home-based PC: A Cochrane review describes four RCTs of home-based PC. The interventions were delivered primarily by multidisciplinary teams including PC nurses and specialists, occupational therapists, physiotherapists, nutritionists, and social work. These home-based specialty models have demonstrated an increase in the number of deaths at home, improved caregiver satisfaction, and reduced overall costs [58]. A review on effectiveness of home PC services for adults with serious illnesses and their caregivers also reported positive outcomes [59]. Notably, it showed increased odds of dying at home (OR 2.21) and reduced symptom burden, without much impact on caregiver grief.

\section{Non-specialists (Primary/Generalist) PC Models}

\section{Primary Palliative Care Models}

Primary PC has been defined as "the clinical management and care coordination including assessment, triage, and referral using a palliative approach for patients with uncomplicated needs associated with a life limiting illness and/or end of life care. Has formal links with a specialist PC provider for purposes of referral, consultation and access to specialist care as necessary." [60] The European Association for Palliative Care used a Delphi process to identify the best strategies to expand PC in primary care throughout Europe. The toolkit stresses that these models will be most successful if generalist professionals are involved from the beginning and that policy, education/training, and implementation (practice) initiatives are all considered $[61,62]$. In the USA there are numerous examples of a focus on primary palliative care. We describe two such models that are being tested and may be applicable in the ALD population.

\section{Integrated Oncology Primary Palliative care (for Lung Cancer)}

Using the NCP Consensus guidelines, a primary PC intervention was developed for lung cancer patients [63]. This intervention consisted of 3 key components: (1) an oncology nurse completed a comprehensive baseline assessment of QOL, symptoms, and psychological distress which was transferred to a care plan, (2) the patient was presented at the interdisciplinary oncology team weekly meeting in which recommendations were presented and approved by the treating oncology team; and (3) patients received 4 educational sessions with content on physical, psychological, social, and spiritual domains of QOL. Outcome measures showed that patients had improved QOL, symptoms, lower psychological distress, higher levels of advance directive completion, and more referrals to supportive care specialists compared to patients receiving usual oncology care. This trial illustrates the importance of attention to multiple system issues when designing primary PC; including access in some way to specialty PC services for complex cases.

\section{PAL LIVER (PALliative Care for End Stage LIVER Disease)}

A large cluster randomized trial (PAL-LIVER, Clinical Trials\# NCT03540771 ) is underway comparing consultative (specialty) PC vs. (primary/generalist) trained hepatologistled PC [64••]. This study involves clinical centers across USA and is currently recruiting. The hepatologists took an online PC training over an average of 12 weeks and deliver the PC intervention. The intervention is to render $\mathrm{PC}$, as taught to hepatologists through an online training and as delivered by PC providers as routine care. The elements of the intervention are guided by a checklist and implemented over the course of interactions with the patient and caregivers at the initial, 1-, 2-, and 3-month visits, to include:

1. Patient/caregiver understanding of diagnosis, illness, and prognosis

2. Symptom assessment and management

3. Psychosocial assessment and management

4. Distress screening and management 
5. Discussion of goals of care

6. Advanced directives

The primary outcome is change in QoL from baseline to 3 months. Secondary outcomes include symptom burden, depression, distress, satisfaction with care, caregiver QoL, and caregiver burden.

Next steps: Future research needs to compare and contrast which elements of these models increase clinical effectiveness and reduce cost and identify the quality metrics for PC services to align within ALD care. Heterogeneity of treatment effects must be considered while considering the effects of PC models. Measuring program successes will be required to demonstrate value and inform sustainability. Metrics such as symptom scores, length of stay, clinical and patient satisfaction, and cost avoidance or cost savings outcomes are important. Hepatology organizations can promote consensusbased integrated PC models that can guide research and practice efforts to increase supportive care for these patients in need and their family caregivers.

\section{Conclusions}

PC has undergone rapid growth within USA. Hospitalbased PC focuses on caring for seriously ill patients. Outpatient clinics, home-based or telehealth-based PC programs have demonstrated feasibility and effectiveness. PC can be delivered by specialists or non-specialists trained in PC. Ongoing research within ALD will inform some elements of a successful model of PC delivery within hepatology; however future research needs to continue to capture evidence on other models from oncology. Due to limited PC workforce, there is a high need to train other healthcare providers with primary PC skills. Aligning healthcare incentive payments with PC services is an important area of future research.

Funding The authors are supported by a Patient-Centered Outcomes Research Institute (PCORI) Award (PLC-1609-36714). The statements in this article are solely the responsibility of the authors and do not necessarily represent the views of the Patient-Centered Outcomes Research Institute (PCORI), its Board of Governors or Methodology Committee.

\section{Declarations}

Human and Animal Rights and Informed Consent This article does not contain any studies with human or animal subjects performed by any of the authors.

Conflict of interest Manisha Verma and Marie A. Bakitas declare that they have no conflict of interest.

\section{References}

Papers of particular interest, published recently, have been highlighted as:

- Of importance

- Of major importance

1. Tapper EB, Parikh ND. Mortality due to cirrhosis and liver cancer in the United States, 1999-2016: observational study. Bmj. 2018;362:k2817.

2. Centers for Disease Control. Health, United States 2017 with special feature on mortality. https://www.cdc.gov/nchs/data/hus/ hus17.pdf (accessed March 16, 2020)

3. Henley SJ, Ward EM, Scott S, Ma J, Anderson RN, Firth AU, et al. Annual report to the nation on the status of cancer, part I: national cancer statistics. Cancer. 2020;126:2225-49.

4. Ferlay J, Colombet M, Soerjomataram I, Mathers C, Parkin DM, Piñeros $\mathrm{M}$, et al. Estimating the global cancer incidence and mortality in 2018: GLOBOCAN sources and methods. Int J Cancer. 2019;144(8):1941-53.

5. Benjamin EJ, Muntner P, Alonso A, Bittencourt MS, Callaway CW, Carson AP, et al. Heart disease and stroke statistics-2019 update: a report from the American heart association. Circulation. 2019;139(10):e56-e528.

6. Peng JK, Hepgul N, Higginson IJ. Symptom prevalence and quality of life of patients with end-stage liver disease: a systematic review and meta-analysis. Palliat Med. 2019;33(1):24-36.

7. Stepanova M, De Avila L, Afendy M, Younossi I, Pham H, Cable $\mathrm{R}$, et al. Direct and indirect economic burden of chronic liver disease in the United States. Clin Gastroenterol Hepatol. 2017;15(5): $759-66$.

8. Xu F, Tong X, Leidner AJ. Hospitalizations and costs associated with hepatitis $\mathrm{C}$ and advanced liver disease continue to increase. Health Aff. 2014;33(10):1728-35.

9. Shrestha D, Rathi S, Grover S, Taneja S, Duseja A, Chawla YK, et al. Factors affecting psychological burden on the informal caregiver of patients with cirrhosis: looking beyond the patient. J Clin Exp Hepatol. 2020;10(1):9-16.

10. Hareendran A, Devadas K, Sreesh S, Tom Oommen T, Varghese J, Lubina S, et al. Quality of life, caregiver burden and mental health disorders in primary caregivers of patients with cirrhosis. Liver Int. 2020;40:2939-49.

11. Nguyen DL, Chao D, Ma G, Morgan T. Quality of life and factors predictive of burden among primary caregivers of chronic liver disease patients. Ann Gastroenterol. 2015;28(1):124.

12. Larson AM. Palliative care for patients with end-stage liver disease. Curr Gastroenterol Rep. 2015;17(5):440. https://doi.org/10.1007/ s11894-015-0440-6.

13. Low JT, Rohde G, Pittordou K, Candy B, Davis S, Marshall A, et al. Supportive and palliative care in people with cirrhosis: international systematic review of the perspective of patients, family members and health professionals. J Hepatol. 2018;69(6):1260-73.

14. Woodrell CD, Hansen L, Schiano TD, Goldstein NE. Palliative care for people with hepatocellular carcinoma, and specific benefits for older adults. Clin Ther. 2018;40(4):512-25. https://doi.org/10. 1016/j.clinthera.2018.02.017.

15. Ufere NN, O'Riordan DL, Bischoff KE, Marks AK, Eneanya N, Chung RT, et al. Outcomes of palliative care consultations for hospitalized patients with liver disease. J Pain Symptom Manag. 2019;58(5):766-73.

16. National Consensus Project. Clinical practice guidelines for quality palliative care. 4th ed. Richmond: National consensus project for quality palliative care; 2018 . 
17. Ferrell BR, Temel JS, Temin S, Alesi ER, Balboni TA, Basch EM, et al. Integration of palliative care into standard oncology care: American society of clinical oncology clinical practice guideline update. J Clin Oncol. 2017;35:96-112.

18. Braun LT, Grady KL, Kutner JS, Adler E, Berlinger N, Boss R, et al. Palliative care and cardiovascular disease and stroke: a policy statement from the American Heart Association/American Stroke Association. Circulation. 2016;134(11):e198-225.

19. Ahluwalia SC, Chen C, Raaen L, Motala A, Walling AM, Chamberlin M, et al. A systematic review in support of the national consensus project clinical practice guidelines for quality palliative care. J Pain Symptom Manag. 2018;56(6):831-70.

20. Quinn KL, Shurrab M, Gitau K, Kavalieratos D, Isenberg SR, Stall NM, et al. Association of receipt of palliative care interventions with health care use, quality of life, and symptom burden among adults with chronic noncancer illness: a systematic review and meta-analysis. JAMA. 2020;324(14):1439-50.

21. Kamal AH, LeBlanc TW, Meier DE. Better palliative care for all: improving the lived experience with cancer. JAMA. 2016;31:2016.

22. Scibetta C, Kerr K, Mcguire J, Rabow MW. The costs of waiting: implications of the timing of palliative care consultation among a cohort of decedents at a comprehensive cancer center. J Palliat Med. 2016;19(1):69-75.

23. Zimmermann C, Riechelmann R, Krzyzanowska M, Rodin G, Tannock I. Effectiveness of specialized palliative care: a systematic review. Jama. 2008;299(14):1698-709.

24. Kavalieratos D, Corbelli J, Zhang D, Dionne-Odom JN, Ernecoff $\mathrm{NC}$, Hanmer J, et al. Association between palliative care and patient and caregiver outcomes: a systematic review and meta-analysis. JAMA. 2016;316(20):2104-14.

25. Hoerger M, Greer JA, Jackson VA, Park ER, Pirl WF, El-Jawahri A, et al. Defining the elements of early palliative care that are associated with patient-reported outcomes and the delivery of end-of-life care. J Clin Oncol. 2018;36(11):1096-102.

26. Esteban JPG, Rein L, Szabo A, et al. Attitudes of liver and palliative care clinicians toward specialist palliative care consultation for patients with end-stage liver disease. J Palliat Med. 2019;22:804-13.

27. Ufere NN, Donlan J, Waldman L, Dienstag JL, Friedman LS, Corey KE, et al. Barriers to use of palliative care and advance care planning discussions for patients with end-stage liver disease. Clin Gastroenterol Hepatol. 2019;17:2592-9.

28. Quill TE, Abernethy AP. Generalist plus specialist palliative care — creating a more sustainable model. N Engl J Med. 2013;368(13): 1173-5.

29. Lupu D, Force PMWT. Estimate of current hospice and palliative medicine physician workforce shortage. J Pain Symptom Manag. 2010;40(6):899-911.

30. Quill TE, Abernethy AP. Generalist plus specialist palliative carecreating a more sustainable model. N Engl J Med. 2013;368(13): 1173-5.

31. Verma, M, et al. (2020). Development, feasibility and acceptability of an online course to teach primary palliative care skills to hepatology providers. AASLD annual meeting. This abstract describes the key elements of a training program for hepatologists on primary palliative care skills.

32•. Firth AM, O'Brien SM, Guo P, Seymour J, Richardson H, Bridges $\mathrm{C}$, et al. Establishing key criteria to define and compare models of specialist palliative care: a mixed-methods study using qualitative interviews and Delphi survey. Palliat Med. 2019;33(8):1114-24 This article utilizes rigorous methods to identify the core components to characterize the existing specialist $\mathrm{PC}$ models in United Kingdom, and can serve as the basis for this approach elsewhere.

33. Harrison KL, Kotwal AA, Smith AK. Palliative care for patients with noncancer illnesses. JAMA. 2020;324(14):1404-5.
34••. Brereton L, Clark J, Ingleton C, et al. What do we know about different models of providing palliative care? Findings from a systematic review of reviews. Palliat Med. 2017;31(9):781-97 A systematic review outlines the different models comprehensively. Table 2 demonstrates the heterogeneity in populations, interventions, comparators and outcomes for distinct PC approaches.

35. Weissman DE, Meier DE. Operational features for hospital palliative care programs: consensus recommendations. J Palliat Med. 2008;11:1189-94.

36. National Quality Forum. A national framework and preferred practices for palliative and hospice care quality: A Consensus Report. Washington, DC: National Quality Forum; 2006.

37. Center to Advance Palliative Care. America's care of serious illness: a state-by-state report card on access to palliative care in our nation's hospitals. New York; 2019.

38. Schoenherr LA, Bischoff KE, Marks AK, O'Riordan DL, Pantilat SZ. Trends in hospital-based specialty palliative care in the United States from 2013 to 2017. JAMA Netw Open. 2019;2(12): e1917043.

39. El-Jawahri A, LeBlanc T, VanDusen H, et al. Effect of inpatient palliative care on quality of life 2 weeks after hematopoietic stem cell transplantation: a randomized clinical trial. JAMA. 2016;316: 2094-103.

40. Courtright KR, Madden V, Gabler NB, Cooney E, Small DS, Troxel A, et al. Rationale and design of the randomized evaluation of default access to palliative services (REDAPS) trial. Ann Am Thoracic Soc. 2016;13(9):1629-39.

41. Bagcivan G, Bakitas M, Palmore J, Kvale E, Nichols AC, Howell SL, et al. Looking back, moving forward: a retrospective review of care trends in an academic palliative and supportive care program from 2004 to 2016. J Palliat Med. 2019;22(8):970-6.

42. Smith TJ, Coyne P, Cassel B, Penberthy L, Hopson A, Hager MA. A high-volume specialist palliative care unit and team may reduce in-hospital end-of-life care costs. J Palliat Med. 2003;6(5):699-705.

43. Morrison R, Meier D, Carlson M. The healthcare imperative: lowering costs and improving outcomes, vol. 2011. Washington DC: Institute of Medicine; 2011.

44. Calton BA, Alvarez-Perez A, Portman DG, et al. The current state of palliative care for patients cared for at leading US cancer centers: The 2015 NCCN. Palliative Care Survey. J Natl Compr Cancer Netw. 2016;14:859-66.

45. Zimmermann C, Swami N, Krzyzanowska M, et al. Early palliative care for patients with advanced cancer: a cluster-randomised controlled trial. Lancet. 2014;383:1721-30.

46. Haun MW, Estel S. Ru“ cker G, et al: Early palliative care for adults with advanced cancer. Cochrane Database Syst Rev. 2017;6: CD011129.

47. Calton BA, Alvarez-Perez A, Portman DG, Ramchandran KJ, Sugalski J, Rabow MW. The current state of palliative care for patients cared for at leading US cancer centers: The 2015 NCCN Palliative Care Survey. J Natl Compr Cancer Netw. 2016;14:85966.

48. Dalal S, Bruera S, Hui D, Yennu S, Dev R, Williams J, et al. Use of palliative care services in a tertiary cancer center. Oncologist. 2016;21:110-8.

49. Hui D, Mori M, Watanabe SM, et al. Referral criteria for outpatient specialty palliative cancer care: an international consensus. Lancet Oncol. 2016;17:e552-9.

50. Cohn J, Corrigan J, Lynn J, Meier D, Miller J, Shega J, et al. Community-based models of care delivery for people with serious illness. NAM Perspectives. Discussion Paper. Washington, DC: National Academy of Medicine; 2017. https://nam.edu/ community-based-models-of-care-delivery-for-people-withserious-illness/ (accessed Feb 21, 2021) 
51. Bakitas M, Lyons K, Hegel M, et al. Effects of a palliative care intervention on clinical outcomes in patients with advanced cancer: the Project ENABLE II randomized controlled trial. JAMA. 2009;302(7):741-9.

52•. Bakitas MA, Tosteson TD, Li Z, et al. Early versus delayed initiation of concurrent palliative oncology care: patient outcomes in the ENABLE III randomized controlled trial. J Clin Oncol. 2015;33(13):1438-45 This study compared early vs delayed integration of palliative care, and demonstrated improvement in quality of life and end of life care. Notably, they reported statistically significant benefits on survival and caregiver's depression.

53. Dionne-Odom JN, Azuero A, Lyons KD, Hull JG, Tosteson T, Li $Z$, et al. Benefits of early versus delayed palliative Care to informal family caregivers of patients with advanced cancer: outcomes from the ENABLE III randomized controlled trial. J Clin Oncol. 2015;33(13):1446-52.

54. Calton B, Abedini N, Fratkin M. Telemedicine in the time of coronavirus. J Pain Symptom Manag. 2020;60:e12-4.

55. Humphreys J, Schoenherr L, Elia G, Saks NT, Brown C, Barbour S, et al. Rapid implementation of inpatient telepalliative medicine consultations during COVID-19 pandemic. J Pain Symptom Manag. 2020;60:e54-9.

56. Jiang B, Bills M, Poon P. Integrated telehealth-assisted home-based specialist palliative care in rural Australia: a feasibility study. J Telemed Telecare. 2020:1357633X20966466.

57. Kuntz JG, Kavalieratos D, Esper GJ, Ogbu N Jr, Mitchell J, Ellis $\mathrm{CM}$. Feasibility and acceptability of inpatient palliative care efamily meetings during COVID-19 pandemic. J Pain Symptom Manag. 2020;60:e28-32.
58. Shepperd S, Gonçalves-Bradley DC, Straus SE, et al. Hospital at home: home-based end-of-life care. Cochrane Database Syst Rev. 2016;2:CD009231.

59. Gomes B, Calanzani N, Curiale V, McCrone P, Higginson IJ. Effectiveness and cost-effectiveness of home palliative care services for adults with advanced illness and their caregivers. Cochrane Database Syst Rev. 2013;6.

60. Australian Government. Australian institute of health and welfare. Service provider organisation: level of palliative care service 2007. http://meteor.aihw.gov.au/content/index.phtml/itemId/334508. (accessed nov 8, 2020).

61. Murray SA, Mitchell S, Boyd K, Moine S. Palliative care: training the primary care workforce is more important than rebranding. Bmj. 2019;365:14119.

62. https://www.eapcnet.eu/eapc-groups/reference/primary-care (accessed Nov 6, 2020)

63. Ferrell BR, Sun V, Hurria A, Cristea M, Raz DJ, Kim JY, et al. Interdisciplinary palliative care for patients with lung cancer. J Pain Symptom Manag. 2015;50(6):758-67.

64. Verma M, Kosinski AS, Volk ML, Taddei T, Ramchandran K, Bakitas M, et al. Introducing palliative care within the treatment of end-stage liver disease: the study protocol of a cluster randomized controlled trial. J Palliat Med. 2019;22(S1):34-43 This paper describes the two comparative models of palliative care for patients with end stage liver disease, as a part of the ongoing PAL LIVER trial. The palliative care intervention is based on standard guidelines, and can be used for other larger trials.

Publisher's Note Springer Nature remains neutral with regard to jurisdictional claims in published maps and institutional affiliations. 